\title{
The breast cancer genome and the complexity of different subgroups: what does it all mean?
}

SR Johnston

Professor of Breast Cancer Medicine, The Royal Marsden NHS Foundation Trust, London

TITLE The genomic and transcriptomic architecture of 2,000 breast tumours reveals novel subgroups

AUTHORS Curtis C, Shah SP, Chin SF et al.

JOURNAL Nature 2012; 486:346-52.

\author{
Correspondence to SR Johnston, \\ Department of Medicine \\ The Royal Marsden \\ NHS Foundation Trust \\ Fulham Road, Chelsea \\ London SW3 6JJ, UK \\ tel. $+44(0) 2078082748$ \\ e-mail \\ stephen.johnston@rmh.nhs.uk
}

DECLARATION OF INTERESTS No conflicts of interest declared.

\section{SUMMARY}

Recent progress in understanding breast cancer has come from identifying the various different molecular subgroups that exist within this heterogeneous disease. The authors in this Nature paper studied the genomic and transcriptional alterations that exist in two large series of breast cancers from UK and Canada tumour banks that had prolonged clinical follow-up, using one set as a discovery cohort $(n=997)$ which was then tested in a second independent validation cohort $(n=995)$.' Their unsupervised analysis of DNA-RNA profiles in the breast cancer genome specifically looked at copy number alterations (CNAs) which are a frequent acquisition in somatic breast cancers, in addition to loss of gene expression transcripts that may indicate gene deletions, somatic mutations or gene silencing by methylation.

Using this approach, the authors identified ten subgroups with different and distinct clinical outcomes which were then validated in the second cohort. They discovered at least two novel subgroups. One was a high risk ER+ group with amplification of the IIq I3//4 cis-activating region which may contain some known amplicons that code for driver genes such as CCNDI, as well as others such as EMSY, PAKI and RSFI. Another subgroup with an excellent prognosis was marked by a paucity of CNAs, but had a strong immune/inflammatory signature with trans-acting deletion hotspots associated with a lymphocytic infiltrate and mature $T$ lymphocytes with rearranged TCR loci. Yet another group of the so-called basal cancers harboured chromosome 5 deletions that were associated with alterations in the transcriptional control of cell cycle regulation and genomic/chromosomal instability that promote aneuploidy.

\section{OPINION}

This study by Curtis et al. provides a framework for understanding how gene copy number affects gene expression in breast cancer, and identifies subgroups that warrant further investigation of the key driver mutations/ deletions that may be clinically relevant.' The caveat to the latter aspect is that these patients were diagnosed many years ago and received relatively uniform 'one-size fits all' approach to therapy, namely no adjuvant chemotherapy if ER+ and lymph node negative, no trastuzumab for HER2+ tumours, and chemotherapy if node positive. As such, this type of genomic data can only provide information on prognosis, and not the predictive benefit of individual systemic cytotoxic, endocrine or biological therapies which is how we need to use genomic information for breast cancer decision-making in the future. However, attempts to do this are starting to be published, including the Nature paper by Ellis et al. which relates whole genome analysis in $E R+$ tumours to response/resistance to endocrine therapy with aromatase inhibitors. ${ }^{2}$ In addition to the discovery of different subgroups that may or may not respond to therapy, this applied genomic analysis moves us towards mapping out cellular pathways that are linked to tumour biology and response to therapy, and may identify potential candidate targets that could be 'druggable' within a therapeutic discovery programme.

Breast cancer cells are smart and evolve all the time, stacking up mutations in subclones of cells to circumvent the host and the drugs that we devise to combat the disease.The science of breast cancer is astounding, but 'little by little' modern cancer genomics is starting to unravel its heterogeneity together with its complexity.

\section{REFERENCES}

I Curtis C, Shash SP, Chin SF et al. The genomic and transcriptional architecture of 2,000 breast tumors reveals novel subgroups. Nature 2012; 486:346-52. http://dx.doi.org//0.1038/nature 10983

2 Ellis MJ, Ding L, Shen D et al. Whole-genome analysis informs breast cancer response to aromatase inhibition. Nature 2012; 486:353-60. http://dx.doi.org//0.1038/nature I I I43

\section{Further reading}

I Cancer Genome Atlas Network. Comprehensive molecular portraits of human breast tumors. Nature 2012; 490:6I-70. http:// dx.doi.org/l0.1038/nature I I4I/2

2 Stephens PJ,Tarpey PS, Davies $\mathrm{H}$ et al.The landscape of cancer genes and mutational processes in breast cancer. Nature 20 I 2; 486:400-4. 